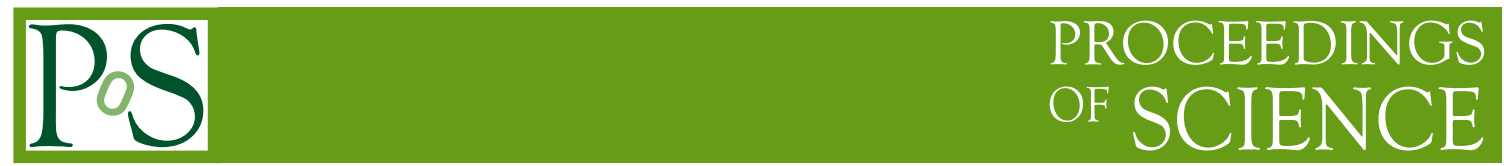

\title{
Heavy flavour production in heavy-ion collisions
}

\section{R. Granier de Cassagnac ${ }^{* \dagger}$}

Laboratoire Leprince-Ringuet, École polytechnique, CNRS/IN2P3, Université Paris-Saclay

E-mail: raphaelecern.ch

\begin{abstract}
We report here on the most recent findings regarding the fate of the heaviest particles when they traverse the hot and dense medium created in heavy-ion collisions, most commonly known as the Quark-Gluon Plasma. We first review the $Z$ and $W$ electroweak bosons, then the heavy quark flavours (charm and beauty) when paired to a light quark (open flavours) or to their own antiquarks (quarkonia).
\end{abstract}

The European Physical Society Conference on High Energy Physics 22-29 July 2015

Vienna, Austria

\footnotetext{
* Speaker.

${ }^{\dagger}$ The author received funding from the European Research Council under the FP7 Grant Agreement no. 259612.
} 


\section{Introduction}

Quantum chromodynamics predicts that hadrons shall melt under extreme conditions of pressure or temperature, liberating their quarks and gluons to form a novel state of matter. This so-called Quark-Gluon Plasma (QGP) is thought to have prevailed during the first microseconds after the big bang, and to be fugitively produced in ultra-relativistic heavy-ion collisions.

This document addresses the physics of the QGP as mined by the heaviest probes. By heavy, we shall mean particles with masses larger than $\Lambda_{Q C D} \simeq 200 \mathrm{MeV}$. These have two interesting properties: a) their total yield is essentially fixed before the QGP is formed (but their kinematics can be strongly modified), and b) their rate and interactions can be calculated via perturbation theory. They include electroweak bosons (section 2) and the heavy quark flavours ( $c$ and $b$ ) when paired to a light quark (open flavours, section 3) or their own antiquarks (quarkonia, section 4). Other probes and aspects of the QGP are reported in another contribution to these proceedings [1].

The first property mentioned above entails that the initial production of these heavy probes is proportional to the number of elementary nucleon-nucleon collisions $\left(N_{\text {coll }}\right)$ occurring in a $\mathrm{PbPb}$ collisions. This suggests the systematic measurement of a nuclear modification factor, $R_{A A}=$ $Y_{A A} / N_{\text {coll }} \times Y_{p p}$, where $Y$ is the yield of a given probe in a heavy-ion $(A A)$ or proton-proton $(p p)$ collision $^{1}$. The $N_{\text {coll }}$ variable, as well as other geometrical quantities such as the number of participating nucleons $\left(N_{\text {part }}\right)$, can be inferred from the events under consideration, thanks to the Glauber model [2]. The variation of $R_{A A}$, in particular with the impact parameter of the collision, is the primary handle to understand the fate of a given probe, and the properties of the QGP. The impact of the collision being not directly accessible, centrality percentiles are defined with multiplicity or energy deposit in detectors that are typically far from the ones used to locate the heavy probe itself. The most central collisions are denoted by $0 \%$.

The Relativistic Heavy-Ion Collider (RHIC) at the Brookhaven National Laboratory (BNL) is providing heavy-ion collisions since 2001. In the late years, it produced a wide range of collisions by varying the beam energy, or the collided species. Among the latest and of particular interest for heavy probe studies are uranium+uranium collisions. The Large Hadron Collider (LHC) at the European Laboratory for Particle Physics (CERN) started in 2010, and delivered about $150 \mu \mathrm{b}^{-1}$ of $\mathrm{PbPb}$ collisions at $\sqrt{s_{\mathrm{NN}}}=2.76 \mathrm{TeV}$, as well as $35 \mathrm{nb}^{-1}$ of $\mathrm{pPb}$ collisions at $\sqrt{s_{\mathrm{NN}}}=5.02 \mathrm{TeV}$. These integrated luminosities correspond to an equivalent number of elementary nucleon-nucleon collisions. While a companion run at $\sqrt{s_{\mathrm{NN}}}=2.76 \mathrm{TeV}$ also provided similar number of pp collisions $\left(5.4 \mathrm{pb}^{-1}\right)$, pp data at $5.02 \mathrm{TeV}$ were missing to serve as reference to the $\mathrm{pPb}$ data at the time of this write-up.

At RHIC, the PHENIX and STAR experiments released a wealth of results on the charm quark, while bottom quarks are much less produced, and electroweak bosons are beyond efficient production thresholds. At LHC, the QGP-dedicated experiment ALICE allowed making extensive studies of charm, while the multi-purpose experiments ATLAS and CMS opened the era of beauty and electroweak studies. The fourth LHCb collaboration also fathered results, limited for now to $\mathrm{pPb}$ collisions.

\footnotetext{
${ }^{1}$ Strictly speaking, a mix of proton-proton and proton-neutron collisions should be used, but with the notable exception of the $W^{ \pm}$bosons, the production modes of the heavy probes do not depend on the isospin of the initial state.
} 


\section{Electroweak bosons}

Electroweak bosons are insensitive to QCD and should thus be unaffected by the QGP. They can thus be considered as standard candles. Their production should then essentially be proportional to the number of elementary nucleon-nucleon collisions occurring in a $\mathrm{PbPb}$ collisions, namely their nuclear modification factor should be equal to unity, $R_{A A}=1$ (taking into account the nucleon content effect in the case of charge-separated $W^{ \pm}$bosons).

In $\mathrm{PbPb}$ collisions at the $\mathrm{LHC}$, this was verified with a typical $10 \%$ precision with both $\mathrm{Z}[3,4]$ and $\mathrm{W}[5,6]$ bosons, by the ATLAS and CMS collaborations. Figure 1 gives two arbitrary examples of these studies: the rapidity dependence of Z boson production in CMS on the left [4], and the centrality dependence of $\mathrm{W}$ boson production in ATLAS on the right [5]. In the later, centrality is depicted by the number of participating nucleons, $N_{\text {part }}$.
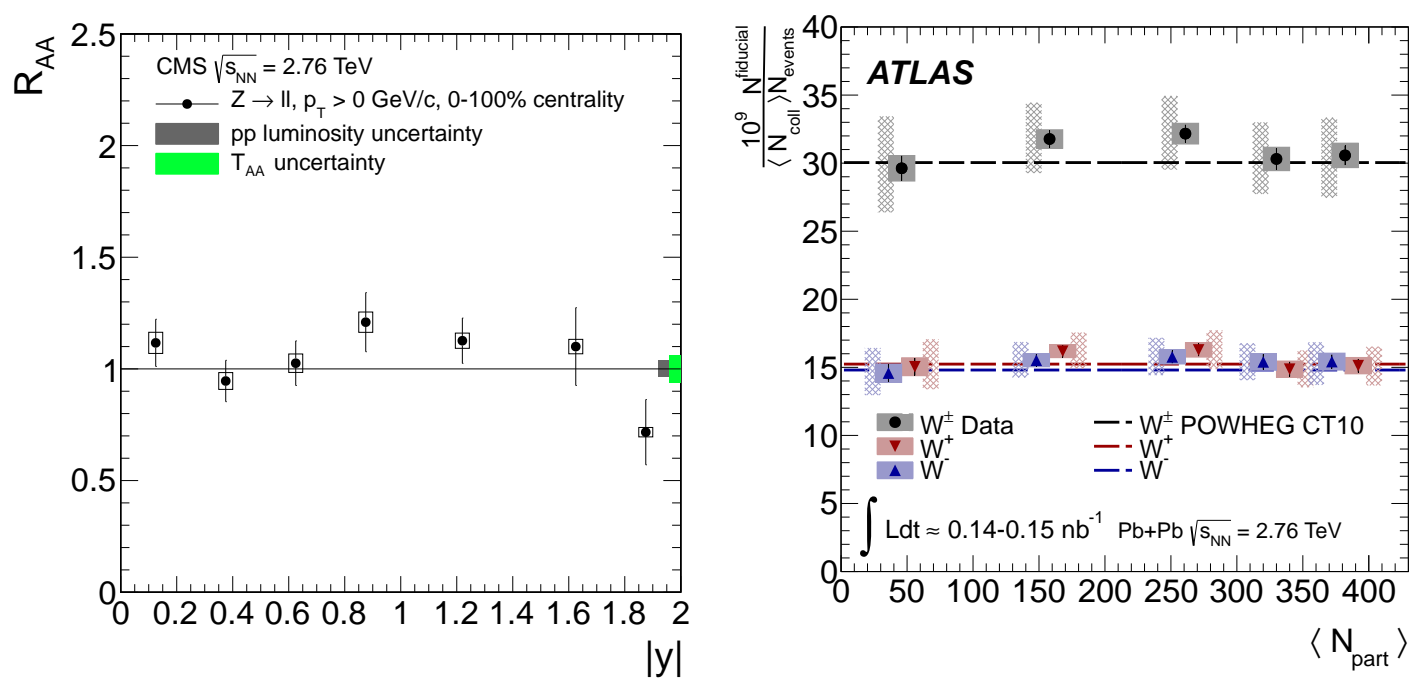

Figure 1: Weak bosons in $\mathrm{PbPb}$ collisions. Left: Nuclear modification factor of $\mathrm{Z}$ bosons versus their rapidity, from CMS [4]. Right: Production yields of $\mathrm{W}$ bosons divided by $N_{\text {coll }}$, versus event centrality depicted by $N_{\text {part }}$, compared to elementary nucleon-nucleon production (POWHEG), from ATLAS [5].

Though electroweak bosons can be considered at first order as standard candles, their production can be slightly changed in heavy-ion collisions, in particular by modifications of parton distribution function (PDF) in the nuclei. While these modifications are beyond the precision of the $\mathrm{PbPb}$ results, hints are seen in the $\mathrm{pPb}$ collisions, thanks to slightly higher statistics and the collision asymmetry. Indeed, the fact that only one ion is involved in the collision allows spanning a wider range of parton momentum fraction $(x)$ without mixing two of them. The key observable in $\mathrm{pPb}$ collisions becomes the (pseudo)rapidity dependence of the production yields. Figure 2 shows two of these dependencies.

On the left, ATLAS sees a backward/forward asymmetry of the $\mathrm{Z}$ boson production yield [7]. On the right, CMS witnesses a departure of the charged asymmetry of leptons arising from $W^{ \pm}$ decays, $\left.\left(N_{l}^{+}-N_{l}^{-}\right) /\left(N_{l}^{+}+N_{l}^{-}\right)\right)$, in the backward region [8]. The latter could reflect different 
modifications of the up and down quarks. Both these results set constraints on nuclear PDF and should be included in global fits.
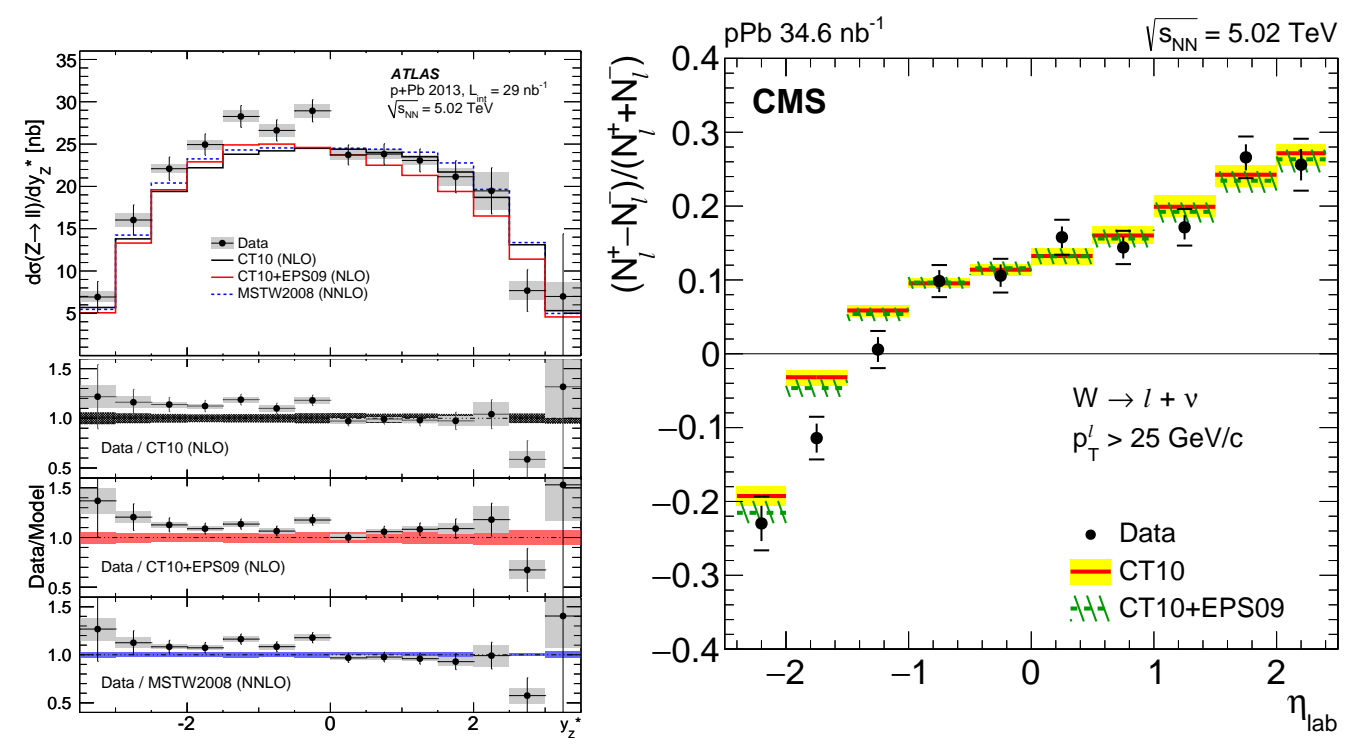

Figure 2: Weak bosons in $\mathrm{pPb}$ collisions. Left: $\mathrm{Z}$ bosons nuclear modification factor versus $\mathrm{Z}$ rapidity, from ATLAS [7]. Right: W charge asymmetry versus lepton pseudorapidity, from CMS [8].

In addition to weak bosons, photons can also be used in the same spirit, but their measurements suffer from larger uncertainties. Such measurements were carried out by the CMS [9] and ATLAS [10] collaborations.

\section{Charm and beauty}

At RHIC, the fate of the charm quark was early settled, with total charm cross section showing binary scaling, $R_{A A}=1$, though with relatively large uncertainties (about $25 \%$ ). This could be measured via single electrons dominated by charm semi-leptonic delay by the PHENIX collaboration [11, 12], then via $D \rightarrow K \pi$ decays from the STAR collaboration [13]. Though the total production cross section of charm quarks is scaling with elementary NN collisions, their dynamics are strongly modified. The early-observed dominant effect is a solid energy loss, that is lowering dramatically the energy (or transverse momentum, $p_{T}$ ) of the outgoing charmed hadrons. The STAR measurement later revealed a more complicated pattern, with the lowest $p_{T} \mathrm{D}$ mesons being slightly depleted $\left(R_{A A}<1\right)$, something that could be understood for instance as the heritage of enhanced flow on the light quark side (see references in [13]). These are depicted as orange squares on Fig. 3, left. They are compared to similar measurements at LHC energies, from the ALICE collaboration $[14,15]$.

Though the first $\mathrm{GeV} / c$ of transverse momentum is missing, the total D meson yield seems suppressed at LHC. Nuclear effects such as PDF modification could contribute to this suppression. It is also to be noted that the charmed hadrons are not all measured and that the charm quarks could be redistributed among them. Measurements of the $D_{S}^{+}$are for instance limited down to 
$p_{T}=4 \mathrm{GeV} / c$, but show a statistically limited hint of lower suppression, with respect to the $D^{0}$, $D^{ \pm}$or $D^{*}[16]$.

As shown on Fig. 3 (right), $D$ mesons appears to be unmodified in $\mathrm{pPb}$ collisions [17], which reinforces the interpretation of the $\mathrm{PbPb}$ suppression in terms of energy loss.
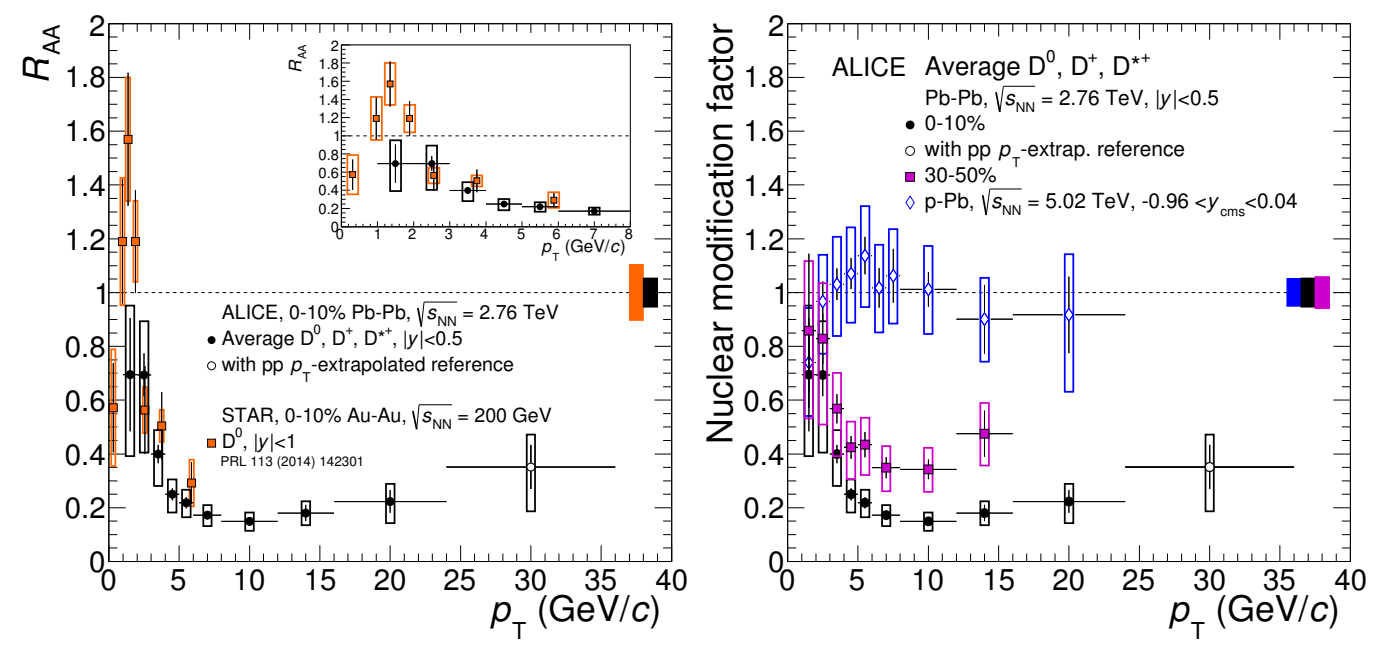

Figure 3: Nuclear modification factor of $\mathrm{D}$ mesons, as a function of $p_{T}$ and for the $10 \%$ most central collisions at LHC, from the ALICE collaboration (black circles from [15]). Left: compared to similar measurement from STAR (orange squares from [13]). Right: compared to the 30-50\% more peripheral $\mathrm{PbPb}$ collisions (purple squares) and $\mathrm{pPb}$ collisions (blue diamonds from [17]).

Another interesting behaviour of charm is its azimuthal anisotropy, that was observed both at RHIC [12] and LHC [15]. More charm is found along the axis containing the centres of the nuclei than in the perpendicular direction. This is interpreted as a collective motion of quarks and gluons, including the heavy charm quark, producing the so-called elliptic flow.

The two above-mentioned phenomena, energy loss (aka jet quenching) and azimuthal anisotropy (aka elliptic flow), show that charm quarks are heavily affected by the QGP. Quantitative and simultaneous description of the two effects, however, is difficult to reach in the various theoretical approaches.

On the $b$-quark side, measurements only became feasible with LHC, thanks to enhanced cross section and luminosity. In $\mathrm{PbPb}$ collisions, a first measurement was made via non-prompt $J / \psi$ (coming from $b$-hadron decays) by CMS [18]. It shows a lower suppression than ALICE's $D$ mesons [19], as shown in Fig. 4 (left). It is to be noted that this does not necessarily mean that $b$ quarks lose less energy than $c$ quarks in the QGP. Indeed, the magnitude of $R_{A A}\left(p_{T}\right)$ being lower than unity does not depend only on the lost energy, but also on the initial quark spectrum and on its fragmentation, not to mention the proportion of quarks coming from gluon splitting. However, the most modern theoretical predictions, referred in [15], include these subtleties, and indeed indicate that charm lose more energy than beauty. An example of such prediction is shown on Fig. 4 (left).

The other measurement directly related to the $b$-quark fate in the QGP is a selection of $b$-quark jets, made by the CMS collaboration, essentially based on the existence of a displaced vertex within 
the jet [20]. The $R_{A A}$ of those, shown on Fig. 4 (right), is roughly half, and compatible with the one of inclusive jets.
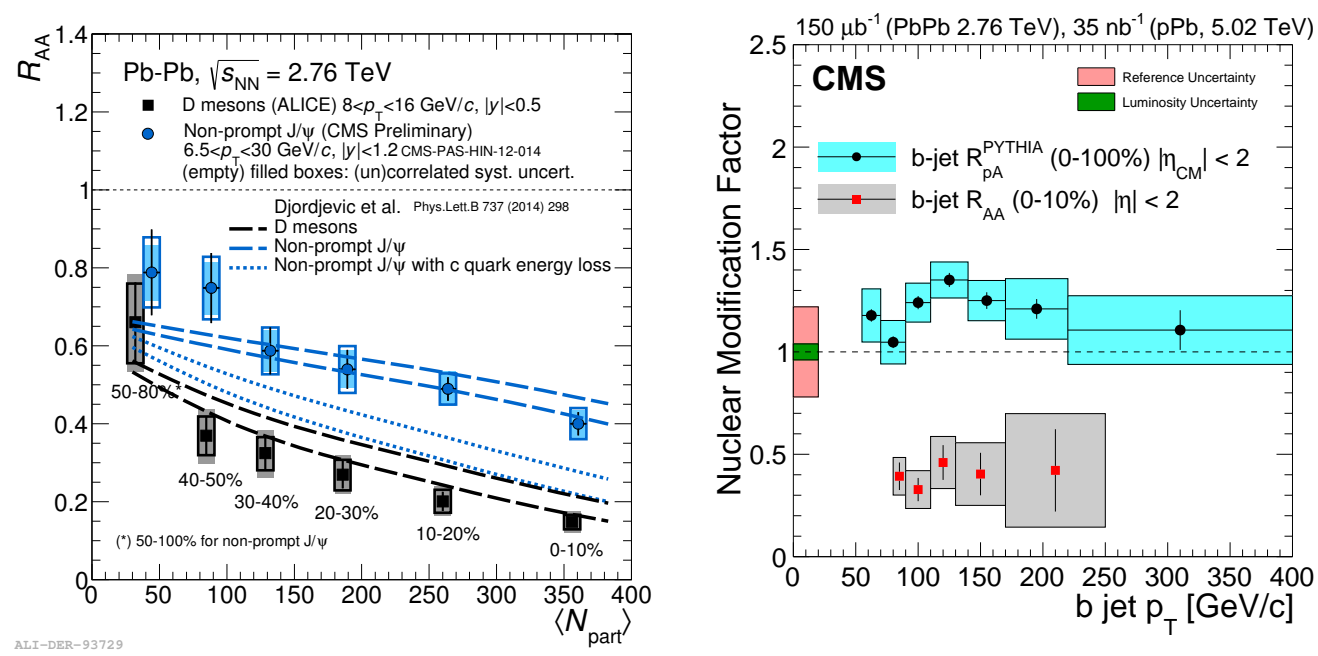

Figure 4: Nuclear modification factor of $b$-quark-related objects. Left: Non-prompt $J / \psi$ from the CMS collaboration (blue circles from the updated but preliminary version of [18]), as a function of centrality depicted by $N_{\text {part }}$, and compared to D mesons from ALICE (black squares from [19]). Right: Jets from b quarks from the $\mathrm{CMS}$ experiment, in $\mathrm{PbPb}$ (red circles from [20]) and $\mathrm{pPb}$ (black circles from [21]).

All the above measurements were also performed in $\mathrm{pPb}$ collisions, showing that no strong suppression affects the $c$ and $b$ quarks. This reinforces the interpretation of the $\mathrm{PbPb}$ collisions as due to a final state effects: energy loss (jet quenching) in the QGP. Those measurements are: D mesons from ALICE [17], non-prompt $J / \psi$ from ATLAS [22] and LHCb [23], and $b$ jets from CMS [21]. They all somewhat suffer from the lack of pp reference at $5 \mathrm{TeV}$.

\section{Quarkonia}

Quarkonium disappearance in heavy-ion collisions $\left(R_{A A}<1\right)$, is one of the earliest and most famous proposed signatures of the QGP. The most accessible quarkonium is the $J / \psi$ meson, via its dilepton decay. As shown on Fig. 5 (left), the ALICE collaboration measured a rather mild drop $\left(R_{A A} \simeq 0.6\right)[24,25]$, while PHENIX had a very strong suppression $\left(R_{A A} \simeq 0.2\right)[26,27]$ in a similar (forward) rapidity range. The current interpretation of this feature lies in the hypothesis that $J / \psi$ can be produced at the final reconfinement step of the QGP evolution, by combination of $c$ and $\bar{c}$ quarks that were not originally created together. Indeed, about a hundred $c \bar{c}$ pairs are produced in a central $\mathrm{PbPb}$ collisions at LHC. Such a regeneration of the $J / \psi$ yield can be seen as a reconfinement, necessarily following a deconfinement.

The regeneration mechanism should also produce low- $p_{T} J / \psi$, and their $R_{A A}$ is indeed seen to decrease as a function $p_{T}$, as shown by ALICE in Ref. [25] and on Fig. 5 (right), and up to the highest $p_{T}$ explored by CMS [18]. Another expected characteristic of the regenerated $J / \psi$ is that they should inherit the elliptic flow of the charm quarks, and the ALICE experiment indeed reported a hint of a positive azimuthal anisotropy $\left(v_{2}\right)$ [28]. At higher $p_{T}$, the CMS collaboration also reported 
a preliminary result showing a positive $v_{2}$ of similar magnitude, though both measurements suffer from large uncertainties.
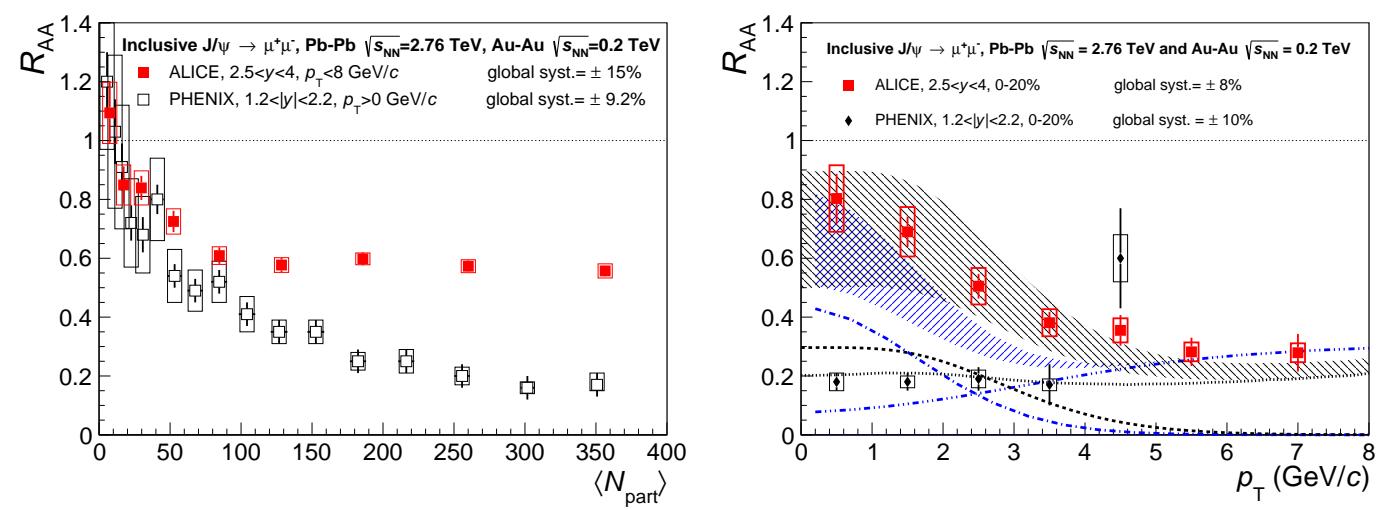

Figure 5: Nuclear modification factors of the $J / \psi$ mesons from the ALICE collaboration at LHC (full red squares from [25]) and the PHENIX collaboration at RHIC (empty squares from [27]), in a similar rapidity range. Left: as a function of centrality depicted by $N_{\text {part }}$. Right: as a function of $p_{T}$ for the $20 \%$ most central events.

In $\mathrm{pPb}$ collisions at LHC, some suppression is observed in the forward (p-going) direction (correspond to the lower parton momentum fractions $x$ ), by ALICE [29], ATLAS [22] and LHCb [23]. This suppression is qualitatively reproduced by nuclear PDF modifications. The same was qualitatively observed at RHIC by PHENIX [30].

Moving to higher quarkonium states, interesting measurements of the $\psi(2 S)$ were released by the CMS [31] and ALICE [25] collaboration. These measurements are summarised on Fig. 6 (left). At high $p_{T}$, the $\psi(2 S)$ appears to be more suppressed than the $J / \psi$ (blue squares from [31]). At lower $p_{T}$, the situation is less clear, and the results statistically limited, from both collaborations (red squares from [31] and purple and black points or limits from [25]).

Concerning bottomonia, CMS early observed the sequential suppression of the three $\Upsilon(1 S)$, $\Upsilon(2 S)$ and $\Upsilon(3 S)$ states [32,33]. The $\Upsilon(3 S)$ is indeed unobserved $\left(R_{A A}(3 S)<0.14\right.$ with a $95 \%$ confidence level), while $R_{A A}(1 S)=0.56 \pm 0.08$ (stat.) \pm 0.07 (syst.) and $R_{A A}(2 S)=0.12 \pm 0.04($ stat. $) \pm$ 0.02 (syst.). ${ }^{2}$ The centrality dependence of the $\Upsilon(1 S)$ and $\Upsilon(2 S)$ suppression is reported on Fig. 6 (right). It is compared to recent $\Upsilon(1 S)$ measurement from STAR at RHIC [34], including preliminary results from UU collisions that reach a similar amount of suppression, in the most central collisions. While CMS released a preliminary result showing that the $\Upsilon(1 S)$ suppression is essentially independent of its $p_{T}$ (up to $20 \mathrm{GeV} / c$ ), measurements made by ALICE complement the reach in rapidity up to 4 and might point to an increase of the suppression in the forward region [35].

Last, it is worth mentioning that whenever they were looked at in $\mathrm{pPb}$-like collisions, the excited states $(\psi(2 S), \Upsilon(2 S), \Upsilon(3 S))$ were seen to be more affected than their corresponding ground states $(J / \psi$ and $\Upsilon(1 S))$. This was for instance the case for the $\psi(2 S)$ at RHIC [36] or LHC [37], and the $\Upsilon(2 S)$ and $\Upsilon(3 S)$ at LHC [38]. The event-multiplicity dependence of these excited/ground

\footnotetext{
${ }^{2}$ It is to be noted that the observed $\Upsilon(1 S)$ are not all directly produced, but that a large fraction of them (around $50 \%$ ) comes from the decay of excited states. Consequently, the real directly produced $\Upsilon(1 S)$ could still be surviving the QGP, justifying their $R_{A A} \simeq 0.5$.
} 

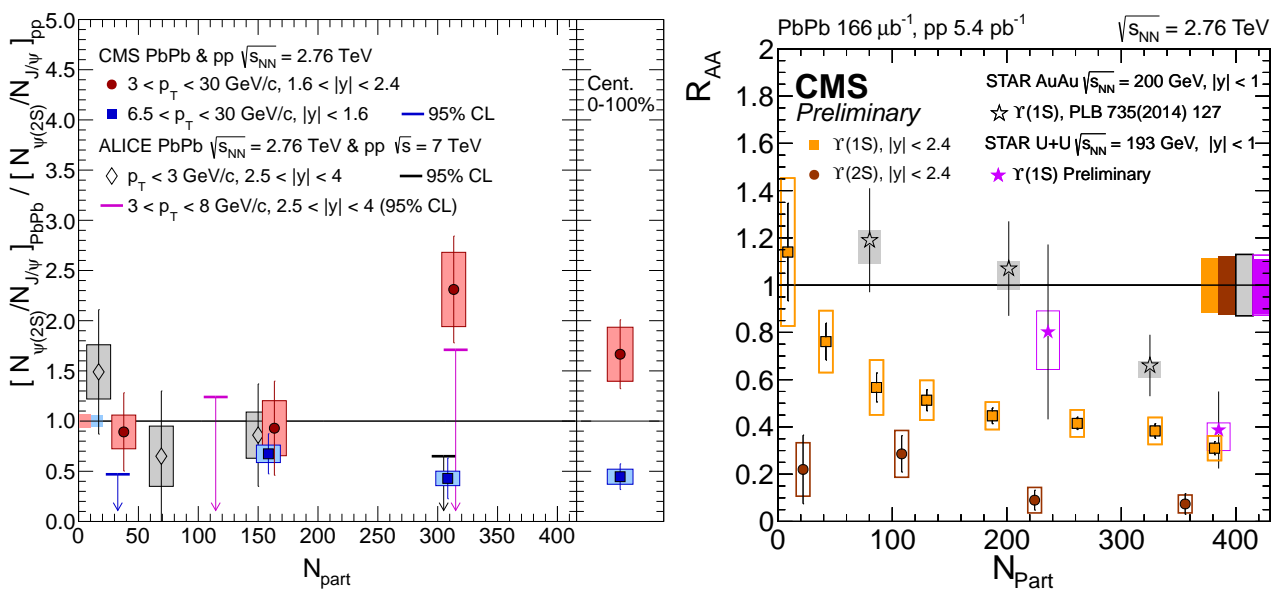

Figure 6: Quarkonium suppression as a function of centrality depicted by $N_{\text {part }}$. Left: Of the $\psi(2 S)$ relative to the $J / \psi$, in various ranges of rapidity and $p_{T}$, from CMS [31] and ALICE [25]. Right: Of the $\Upsilon(1 S)$ at LHC (orange squares from [32]) and RHIC (stars from [35]), and of the $\Upsilon(2 S)$ (red circles from [32]).

state ratio in particular is highly non-trivial to understand and reveal a strong interplay between the environment and the excited states.

\section{Summary}

In summary, the heaviest particles revealed interesting properties related to the Quark-Gluon Plasma.

The electroweak bosons are good candles and they start to reveal subtle initial-state nuclear effects, at the $10 \%$ level, typically. As in more elementary collisions, they serve as a reference process.

The heavy quarks, charm and beauty, are affected by the two striking modifications of the lighter flavours: energy loss (jet quenching) and collective motion (elliptic flow). The magnitude of these effects are of crucial importance for theorists to understand the underlying interaction mechanisms occurring within the QGP.

When bound together in quarkonia, they show a complicated pattern of suppression, a rich phenomenology of effects, among which a probable regeneration of charmonia at LHC. Bottomonia show a sequential suppression pattern, which is as close as one can hope to the dreamt QGP thermometer.

\section{References}

[1] Bielcikova, J., Jets and correlations in heavy-ion collisions, these proceedings.

[2] Miller, M. L. and Reygers, K. and Sanders, S. J. and Steinberg, P., Glauber modeling in high energy nuclear collisions, Ann. Rev. Nucl. Part. Sci. 57 (2007) 205, [nucl-ex/ 0701025 ].

[3] ATLAS Collaboration, Measurement of $Z$ boson Production in Pb+Pb Collisions at $\sqrt{s_{N N}}=2.76 \mathrm{TeV}$ with the ATLAS Detector, Phys. Rev. Lett. 110 (2013) 022301, [arXiv:1210 . 6486]. 
[4] CMS Collaboration, Study of Z production in $\mathrm{PbPb}$ and pp collisions at $\sqrt{s_{\mathrm{NN}}}=2.76 \mathrm{TeV}$ in the dimuon and dielectron decay channels, JHEP 03 (2015) 022, [arXiv: 1410 . 4825].

[5] ATLAS Collaboration, Measurement of the production and lepton charge asymmetry of W bosons in $P b+P b$ collisions at $\sqrt{s_{N N}}=2.76$ TeV with the ATLAS detector, Eur. Phys. J. C75 (2015) 23, [arXiv:1408.4674].

[6] CMS Collaboration, Study of W boson production in PbPb and pp collisions at $\sqrt{s_{N N}}=2.76 \mathrm{TeV}$, Phys. Lett. B715 (2012) 66, [arXiv:1205.6334].

[7] ATLAS Collaboration, $Z$ boson production in $p+P b$ collisions at $\sqrt{s_{N N}}=5.02$ TeV measured with the ATLAS detector, Phys. Rev. C92 (2015) 044915, [arXiv: 1507 . 06232 ].

[8] CMS Collaboration, Study of W boson production in pPb collisions at $\sqrt{s_{\mathrm{NN}}}=5.02$ TeV, Phys. Lett. B750 (2015) 565, [arXiv:1503.05825].

[9] CMS Collaboration, Measurement of isolated photon production in $\mathrm{pp}$ and $\mathrm{PbPb}$ collisions at $\sqrt{s_{N N}}=2.76 \mathrm{TeV}$, Phys. Lett. B710 (2012) 256, [arXiv:1201.3093].

[10] ATLAS Collaboration, Centrality, rapidity and transverse momentum dependence of isolated prompt photon production in lead-lead collisions at $\sqrt{s_{\mathrm{NN}}}=2.76 \mathrm{TeV}$ measured with the ATLAS detector, arXiv:1506.08552.

[11] PHENIX Collaboration, Centrality dependence of charm production from single electrons measurement in $A u+A u$ collisions at $\sqrt{\left.s_{(} N N\right)}=200$ GeV, Phys. Rev. Lett. 94 (2005) 082301, [nucl-ex/0409028].

[12] PHENIX Collaboration, Heavy Quark Production in $p+p$ and Energy Loss and Flow of Heavy Quarks in $A u+A u$ Collisions at $\sqrt{s_{N N}}=200$ GeV, Phys. Rev. C84 (2011) 044905, [arXiv:1005.1627].

[13] STAR Collaboration, Observation of $D^{0}$ Meson Nuclear Modifications in Au+Au Collisions at $\sqrt{s_{N N}}=200 \mathrm{GeV}$, Phys. Rev. Lett. 113 (2014), no. 14 142301, [arXiv: 1404.6185 ].

[14] ALICE Collaboration, Suppression of high transverse momentum $\mathrm{D}$ mesons in central Pb-Pb collisions at $\sqrt{s_{N N}}=2.76 \mathrm{TeV}$, JHEP 09 (2012) 112, [arXiv: 1203.2160 ].

[15] ALICE Collaboration, Transverse momentum dependence of D-meson production in Pb-Pb collisions at $\sqrt{s_{\mathrm{NN}}}=2.76 \mathrm{TeV}, \operatorname{arXiv}: 1509.06888$.

[16] ALICE Collaboration, Adam, Jaroslav and others, Measurement of $D_{s}^{+}$production and nuclear modification factor in $\mathrm{Pb}$-Pb collisions at $\sqrt{s_{\mathrm{NN}}}=2.76 \mathrm{TeV}$, arXiv:1509.07287.

[17] ALICE Collaboration, Measurement of prompt D-meson production in $p$-Pb collisions at $\sqrt{s_{N N}}=$ 5.02 TeV, Phys. Rev. Lett. 113 (2014), no. 23 232301, [arXiv: 1405.3452 ].

[18] CMS Collaboration, Suppression of non-prompt $J / \psi$, prompt $J / \psi$, and $Y(1 S)$ in PbPb collisions at $\sqrt{s_{N N}}=2.76 \mathrm{TeV}, \mathrm{JHEP} 05$ (2012) 063, [arXiv: 1201.5069 ]. [Preliminary updated analysis in CMS PAS HIN-12-014].

[19] ALICE Collaboration, Centrality dependence of high- $p_{\mathrm{T}} \mathrm{D}$ meson suppression in Pb-Pb collisions at $\sqrt{s_{\mathrm{NN}}}=2.76 \mathrm{TeV}, \operatorname{arXiv}: 1506.06604$.

[20] CMS Collaboration, Evidence of b-Jet Quenching in PbPb Collisions at $\sqrt{s_{N N}}=2.76$ TeV, Phys. Rev. Lett. 113 (2014) 132301, [arXiv: 1312 . 4198]. [Erratum: Phys. Rev. Lett.115,029903(2015)].

[21] CMS Collaboration, Transverse momentum spectra of b jets in pPb collisions at $\sqrt{s_{N N}}=5.02 \mathrm{TeV}$, arXiv:1510.03373. 
[22] ATLAS Collaboration, Measurement of differential $J / \psi$ production cross sections and forward-backward ratios in p+Pb collisions with the ATLAS detector, Phys. Rev. C92 (2015) 034904, [arXiv:1505.08141].

[23] LHCb Collaboration, Study of J/ $\psi$ production and cold nuclear matter effects in pPb collisions at $\sqrt{s_{N N}}=5 \mathrm{TeV}$, JHEP 02 (2014) 072, [arXiv: 1308 . 6729].

[24] ALICE Collaboration, $J / \psi$ suppression at forward rapidity in $\mathrm{Pb}$ - $\mathrm{Pb}$ collisions at $\sqrt{s_{N N}}=2.76 \mathrm{TeV}$, Phys. Rev. Lett. 109 (2012) 072301, [arXiv:1202.1383].

[25] ALICE Collaboration, Differential studies of inclusive $J / \psi$ and $\psi(2 S)$ production at forward rapidity in Pb-Pb collisions at $\sqrt{s_{\mathrm{NN}}}=2.76 \mathrm{TeV}$, arXiv:1506.08804.

[26] PHENIX Collaboration, $J / \psi$ Production vs Centrality, Transverse Momentum, and Rapidity in Au+Au Collisions at $\sqrt{s_{N N}}=200$ GeV, Phys. Rev. Lett. 98 (2007) 232301, [nucl-ex/ 0611020 ].

[27] PHENIX Collaboration, $J / \psi$ suppression at forward rapidity in Au+Au collisions at $\sqrt{s_{N N}}=200 \mathrm{GeV}$, Phys. Rev. C84 (2011) 054912, [arXiv:1103.6269].

[28] ALICE Collaboration, $J / \psi$ Elliptic Flow in Pb-Pb Collisions at $\sqrt{s_{\mathrm{NN}}}=2.76$ TeV, Phys. Rev. Lett. 111 (2013) 162301, [arXiv:1303.5880].

[29] ALICE Collaboration, Rapidity and transverse-momentum dependence of the inclusive $J / \psi$ nuclear modification factor in $p$-Pb collisions at $\sqrt{s_{N N}}=5.02 \mathrm{TeV}$, JHEP 06 (2015) 055, [arXiv:1503.07179].

[30] PHENIX Collaboration, Cold Nuclear Matter Effects on J/Psi as Constrained by Deuteron-Gold Measurements at $s(N N) * *(1 / 2)=200-G e V$, Phys. Rev. C77 (2008) 024912, [arXiv: 0903.4845$].$ [Erratum: Phys. Rev.C79,059901(2009)].

[31] CMS Collaboration, Measurement of Prompt $\psi(2 S) \rightarrow J / \psi$ Yield Ratios in Pb-Pb and p-p Collisions at $\sqrt{s_{N N}}=2.76$ TeV, Phys. Rev. Lett. 113 (2014) 262301, [arXiv:1410.1804].

[32] CMS Collaboration, Observation of sequential Upsilon suppression in PbPb collisions, Phys. Rev. Lett. 109 (2012) 222301, [arXiv:1208.2826].

[33] CMS Collaboration, Indications of suppression of excited $\Upsilon$ states in PbPb collisions at $\sqrt{s_{N N}}=2.76$ TeV, Phys. Rev. Lett. 107 (2011) 052302, [arXiv: 1105.4894 ].

[34] STAR Collaboration, Suppression of $\Upsilon$ production in $d+A u$ and $A u+A u$ collisions at $\sqrt{s_{N N}}=200 \mathrm{GeV}$, Phys. Lett. B735 (2014) 127, [arXiv:1312 . 3675]. [Erratum: Phys. Lett.B743,537(2015)].

[35] ALICE Collaboration, Suppression of $\Upsilon(1 S)$ at forward rapidity in $\mathrm{Pb}-\mathrm{Pb}$ collisions at $\sqrt{s_{\mathrm{NN}}}=2.76 \mathrm{TeV}$, Phys. Lett. B738 (2014) 361, [arXiv: 1405.4493 ].

[36] PHENIX Collaboration, Nuclear Modification of $\psi^{\prime}, \chi_{C}$, and $J / \psi$ Production in $d+A u$ Collisions at $\sqrt{s_{N N}}=200 \mathrm{GeV}$, Phys. Rev. Lett. 111 (2013), no. 20 202301, [arXiv:1305.5516].

[37] ALICE Collaboration, Suppression of $\psi(2 S)$ production in p-Pb collisions at $\sqrt{s_{\mathrm{NN}}}=5.02 \mathrm{TeV}$, JHEP 12 (2014) 073, [arXiv: 1405.3796 ].

[38] CMS Collaboration, Event activity dependence of $Y(n S)$ production in $\sqrt{s_{N N}}=5.02 \mathrm{TeV} p P b$ and $\sqrt{s}=2.76 \mathrm{TeV}$ pp collisions, JHEP 04 (2014) 103, [arXiv : 1312 . 6300 ]. 